\title{
Research on Voltage and Reactive Power Integrated Automatic Control System in Substation
}

\author{
Zhenyuan $\mathrm{Fu}^{1, \mathrm{a}}$ \\ ${ }^{1}$ School of Automation, Wuhan University of Technology, \\ Wuhan, 430070, China
}

Keywords: Substation; Voltage and Reactive Power Integrated Control; System Analysis

\begin{abstract}
With the continuous development and progress of modern economic society, the power system began to occupy an increasingly important position in all walks of life, the conventional substation management mode cannot meet the needs of related work, so be able to learn from and use substation synthesis automation technology. Under the influence of the continuous development and progress of the national economy, people's daily production and life began to put forward higher and higher demands on the quality of power, how can we ensure that more quality electricity is obtained, and how to further improve the economy of the power grid, as power supply enterprises, power generation enterprises, power distribution companies and related participants need to think and solve the problem. The power quality indicators are divided into harmonic components, voltage amplitude and frequency. Voltage amplitude as an important indicator, the assessment of the object for the voltage of the pass rate; because power consumption and power generation cannot be balanced, so there will inevitably be the phenomenon of voltage fluctuations. The transformer that can carry out the pressure regulator changes the actual number of conductors of the high-voltage side winding to adjust the voltage of the low-voltage side and the medium voltage to adjust the total voltage better. Under the influence of various factors, there are still many problems in the substation reactive power automatic control system. At the same time, the corresponding improvement and improvement measures are put forward to solve these problems in order to create more economic and social benefits.
\end{abstract}

\section{Introduction}

The actual loss of the system is expressed as the actual energy loss of the current through the conductor during transmission. The ideal state of the conductor will not appear no power transmission phenomenon, the actual loss is relatively small. However, the current loss of the grid structure cannot be avoided and you can put the reactor, capacitors and other reactive power to achieve the purpose of balance [1]. Because the user load and the system are constantly changing which requires the adjustment of the relevant equipment to meet the needs of the system pulsation and achieve the energy economy and quality of the operation of the indicators. From this request to analyze, the general choice to change the on-load tap changer tap position, the capacitor in parallel to the reactive and voltage to adjust, so that the voltage pass rate gradually increased, reducing unnecessary losses, so that power grid To meet the needs of power supply quality, so reactive power control system is an important operating equipment.

\section{The Principle Analysis of Voltage and Reactive Power System}

At present, many substations have chosen voltage and reactive power automatic control device, and its type is relatively large. Some units also set the corresponding control devices, including SS-DWK type, VQC-ILA type and 96VQC type, and some are not specifically set, but through the integrated substation automation system integration, the use of various software Module to achieve [2]. If there is only one section of the power supply system, the generator, the on-load tap changer, the equivalent load, the system is equivalent to the voltage source string into the equivalent reactance $\mathrm{L}_{\mathrm{xT}}$ and the ideal transformer carrying the equivalent impedance of $\mathrm{R}+\mathrm{jX}$ load, as shown in the following figure: 


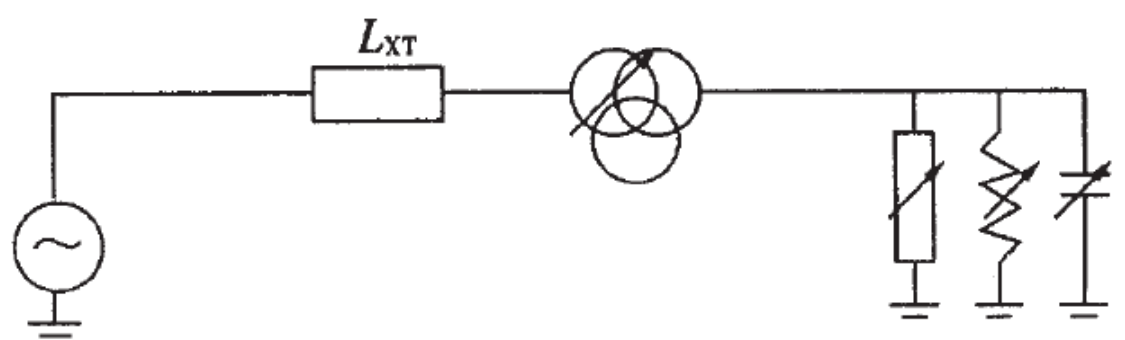

Figure 1 Simple power supply system diagram

The Impact of Reactive Power on the System. Since the load model of R cannot be clarified, if $\mathrm{R}$ has little effect on the voltage as the voltage change $\Delta \mathrm{R}$ can be achieved, the imaginary part of the impedance is infinite when $\mathrm{L}$ and $\mathrm{C}$ can be fully compensated. At this time the specific size of the impedance of the $\mathrm{R}$, the load voltage and the system impedance of the pressure drop. If the inductive load gradually increases, then the imaginary part gradually decreases from positive infinity, taking into account the influence of the limit factor, the imaginary part is close to 0 is infinitely small, when the actual impedance of the size of 0 [3]; load voltage will be close to 0 of the negative infinitescence; taking into account the impact of the limit factor, the imaginary part of the infinitely close to 0 infinitely small, the load voltage will be close to 0 , so the first capacitor is not in the kernel and the environment can lift the voltage, because the voltage $U$ is a continuous function of $\mathrm{X}$, the power factor gradually increased, assuming that the system equivalent reactance $\mathrm{L}_{\mathrm{XT}}$ and $\mathrm{R}$ unchanged, if $\mathrm{Q}$ did not achieve the inversion, the voltage will increase monotonically.

If the generator running in the reactive environment, the excitation current will be gradually reduced, thereby increasing the power angle of the generator, so that the stability of the system gradually reduced, induced resonance over voltage phenomenon. Especially for the local optimization of the phenomenon, because it is difficult to obtain the system of reactive power, if there is a reverse of the reactive power substation, the sum of more than the system reactive load, then the generator cannot run smoothly [4]. Therefore, if the use of capacitors as a specific voltage regulator method, you need to comply with the principle of not to the system reactive power.

The Effect of Voltage on Reactive Power. The reactive load model $Q=a U^{2}+b U+c$, where a is the inductive component, b is the current component, and c is the constant. Q shows the $\mathrm{U}$ function, the voltage increases, reactive power began to increase; voltage decreases, then the reactive power reduction. The relationship between reactive power and voltage and the relationship between active and voltage will have a direct effect on the power factor. Power factors in different load, the emergence of changes are also different. From the above study, it is not difficult to find that there are differences in the reactive power characteristics of the different loads, and the load composition cannot be quantitatively analyzed for different load changes. Therefore, the voltage and power control need to be controlled by the voltage and power factors. Not the reactive as a specific control target.

For the substation, in order to allow reactive power and voltage to achieve the actual demand, can be on the main transformer on-load tap-changer, switching capacitor bank and other methods to substation reactive power and voltage to adjust. Adjust the main transformer on-load tap-changer In addition to the impact on the voltage and there is a significant impact on the reactive [5]. Adjust the main transformer on-load tap-changer and switching capacitor group on the reactive power and voltage is the main basis for the control of voltage and reactive power, the following for the adjustment of the main transformer on-load tap-changer and switching capacitor group Voltage and reactive power trends to be studied.

Because voltage and reactive power have the impact between each other, the voltage and reactive power synthesis control is the scope of choice, from the user's needs to analyze the following several.

First for the voltage priority, voltage and reactive power cannot meet the requirements, you need to ensure that the voltage required to meet the normal requirements; followed by reactive power priority, voltage and reactive power cannot meet the relevant requirements, will be able to meet the normal standard reactive; For the adaptation, voltage and reactive power cannot meet the 
requirements at the same time, to be able to maintain the status quo [6].

At present, many have chosen the voltage priority mode, that is, the UQ plane is divided into nine different regions, combined with different regional voltage level, reactive power, voltage and reactive power control principle to analyze, $Q$ and $U$ values in a certain time After the specified time has elapsed, it is assumed that it has been calculated or analyzed from the empirical value:

Uu indicates that the on-load tap-changer adjusts the maximum amount of change in the control side voltage induced by the first stage;

Uq switching all capacitor-induced control side voltage maximum change;

Qu on-load tap-changer to adjust the maximum amount of reactive power induced by a file;

Qq switching a group of capacitors to induce the maximum amount of reactive power change.

\section{The Adjustment Measures}

Tap position adjustment and capacitor switching control program as shown below, single arrows that the proposed adjustment program, from the above content can be found in the same trend of changes in voltage and reactive power, all belong to adjust the position of the tap position, the opposite trend of the opposite For the switched capacitor group [7]. First of all, even if the switching capacitor has the greatest impact on the reactive power, but still can be the power factor as a specific control target, rather than the reactive as the specific control objectives, $\mathrm{P}$ and $\mathrm{Q}$ can fundamentally reflect the current and voltage of two different physical direction and size. If only the $\mathrm{Q}$ value is used to switch the capacitor, especially in the environment that allows the forward Q, it is difficult to combine the components of P to clear the size of the loss; in the side of the load under the premise of Q and direction and P Different, can be drawn to adjust the target of different control, and the power factor as the actual control objectives, which focus on the two components reflect the $\mathrm{P}$ and $\mathrm{Q}$.

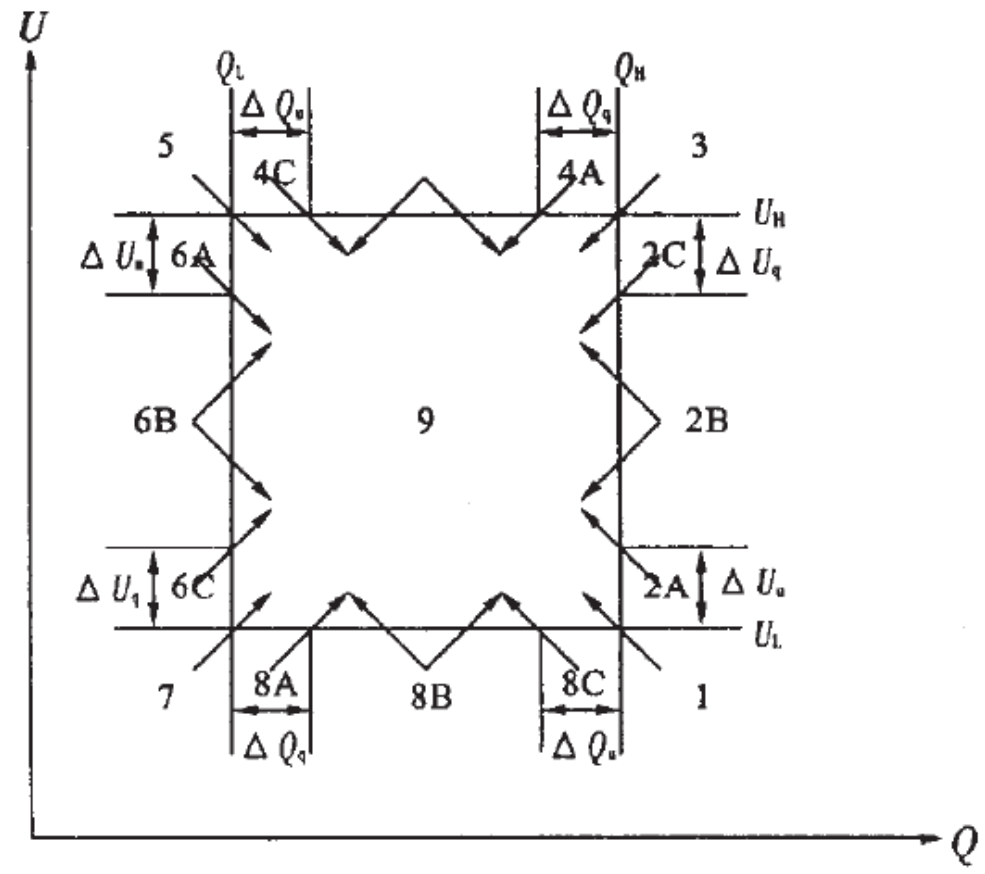

Figure 2 Nine domain map

Second, in the 2B, 4B, 6B and 8B and other areas, it can achieve the adjustment of a total of two programs, both to adjust the tap position, but also on all the capacitor group switching. Because the tap changer is an important component of the transformer, the coil and the transformer core are fixed, the only tap switch can be adjusted when the action. Therefore, the work of the tap-changer can proceed smoothly will directly affect the power supply, if there is pressure failure phenomenon, it may make a regional power outage, to people's production and life caused a greater impact, bring serious The loss [8]. Therefore, it is not scientific to adjust the tap position frequently in the $2 \mathrm{~B}, 4 \mathrm{~B}$ and $6 \mathrm{~B}$ and $8 \mathrm{~B}$ regions, but to be able to give priority to switching the capacitor bank to achieve 
better adjustment. Only when the capacitor cannot be switched, consider the tap position as a practical alternative.

Finally, when the capacitor switching, to be able to follow the principle of cyclic switching, if the choice of circular switching can make the average use of the switch probability is conducive to long-term effective operation of the device. Control measures should also be able to take into account the equipment latch-up related issues, such as TV disconnection, parallel regulator once the main transformer no load, tap rejection, light load, fault and so on, the need for the corresponding equipment, the device group is adjusted for latching.

\section{The Existing Problems and Further Research Direction}

Because the daily load has a peak, valley, equal change, adjust the frequency is relatively high. Sometimes the daily load changes must be able to achieve the adjustment, the current adjustment of the program is the voltage or power factor in the unqualified area after a period of time to adjust, the choice of the actual adjustment strategy is to restore the operational state to the 9th district final goal. Moreover, the overall adjustment of the higher frequency induced the following questions.

Every day there will be electric power factor, the phenomenon of voltage failure, and the total number of changes will be set because of changes in time, this setting value no matter how the settings will be a dilemma. The first to adjust the number of times to induce the healthy operation of the equipment to reduce the level; Second, the failure time is too long to induce low power quality indicators.

As long as the entry into the 9 area after the operation will not exist to adjust the phenomenon, so that the system for a long time running in the power or voltage factor unqualified level, and cannot control the system in the rated operation of the state. Therefore, in order to better solve the relevant problems, we must be able to voltage and reactive power control system to adjust the fuzzy control and intelligent processing, control system to be able to have the ability to self-learning, the system should be able to record in a few days month on the same day, the same day to run the data, make the appropriate action to adjust the record [9]. Which makes it in the running state does not exceed the 9 area, but from the recorded operating data, the recorded adjustment program as a reference, consider the current stage system and rated the best state difference as a general basis, and pre-adjustment, let the system does not run more than 9 areas, and has been in the 9 area to get control of a certain edge, then the voltage and power factor failure gradually reduced, the voltage and reactive power control system cannot be adjusted for the number of times, in order to be able to select the trend mode of operation, Planning optimization to put the corresponding reference value.

Capacitor group switching capacity interval is relatively large, it is difficult to achieve the continuous development of the switching capacity of the development, because the conventional countermeasures set, do not allow the system to send reactive power, and sometimes because of a set of capacitors, That is, do not want to use the system to reverse the capacitor group program, cannot achieve the appropriate adjustment objectives. If you can combine the substation data to the user to control the unified, sub-line scattered on the compensation equipment, you can better solve the relevant problems.

\section{Conclusion}

Substation voltage and reactive power automatic control system through real-time acquisition of substation operating parameters, computer technology, digital signal processing technology, automatic control technology. Automatic control of the main transformer includes compensation capacitors, on-load voltage regulator, so that it is in the best state. This is also to reduce its losses, improve the voltage pass rate and improve the level of safe and economical operation of the main measures which can reduce the labor intensity of duty to create greater economic and social benefits. 


\section{Acknowledgements}

Fund Project: Research on Fault-tolerant Control of a Class of Generalized Systems Based on Robust Adaptive Method(61374151).

\section{References}

[1] Shen Shuming. Realization and discussion of substation voltage and reactive power integrated automatic control [J]. The relay, 2000, 11: 60-62.

[2] Hou Yongquan, Ma cubic; Based on the fuzzy control theory of substation voltage / reactive microcomputer automatic control device [J]. Technology and enterprises, 2015, 06: 73.

[3] Chen Baichao, Song Jieming, Zhou Pan, Ni Xiangping, Zhang Yadi, Yuan Jiayin.Multi-voltage level reactive power and voltage control of $220 \mathrm{kV}$ substation based on MCR and MSVC [J]. Journal of Electrical Engineering, 2015, 16: 230-237.

[4] Zhu Miao. Substation voltage and reactive power integrated automatic control problem [J]. Guangdong Science and Technology, 2013, 16: 82 + 56 .

[5] Zhao Hao. Based on the PLC substation voltage and reactive power integrated control system research[J]. Knowledge economy, 2011, 21: 104

[6] ZHAO Shuo-wei, GUAN Yan-cui.Non-automatic control of substation voltage and reactive power based on ANN [J]. Communication Power Technology, 2014, 02: 71-72.

[7] Zhang Liqin. AVC automatic voltage control system in the smart grid application [J]. China Science and Technology Information, 2016, 16: $44+46$.

[8] Yin Yumin. Discussion on substation voltage reactive power intelligent integrated control strategy [J]. Electronic World, 2016, 16: 76.

[9] Wang Ben, Liu Wenjun, Zhou Xianfei, Lu Yunhe, Xu Sizong, Ma Yue, Cao Weihua. Beijing area automatic voltage control system control strategy [J]. Rural electrification, 2016, 08: 8-10. 